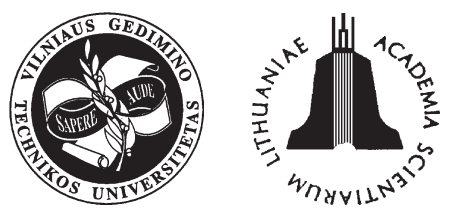

\title{
THE RESEARCH INTO SPEED ABILITY OF FUEL DISPERSION
}

\author{
Dmitry Matievsky¹, Andrey Svistula ${ }^{2}$, Alexander Eskov ${ }^{3}$ \\ Altai State Technical University, 46, Lenin St., 656099 Barnaul, Russia. \\ Tellfax: (+3852260516), Tel: (+3852368475) \\ Received 2004-11-10; accepted 2004-12-03
}

\begin{abstract}
The investigation of the process of carburetion in combustion engines is an actual scientific and practical task. The results of the experimental investigations of the fuel jet flow formed by the nozzle through two section series arranged are shown in the presented article. Front speed and fuel mass speed between optical sections in fuel flow were determined by registered optical density of fuel flow for different pressures. The procedure of the determination of speed abilities of the fuel flow gives the preconditions for creating the bench for the determination of fuel sprayers in the work process of manufacturing with the aim of their division into the groups of one-type parameters. Such approach assumes the usage of manufacturing standard that is the sprayer and the fuel pump with the best ability of injection.
\end{abstract}

Keywords: speed of the flow, process of fuel injection, optical density.

\section{Introduction}

At present 1 the world 2 propulsion 3 engineering uses the best achievements in science and techniques that allow one to improve economic indexes and ecological safety of the engine.

Specific character of fuel burning in the diesel cylinder with great heterogeneity of the fuel-air mixture by proportion and temperature, high values of pressure and temperature predetermines fuel cracking and free carbon evolution as soot with slow delayed burning on the line of broadening parallel to the burning process. That doesn't allow one to use the heat brought with fuel with the most efficiency. Investigations show that the losses of effective use of fuel in the cycle amount to $1-2 \%$ due to the imperfection of the heat input (connected with the soot formation) and $8 \%$ due to the heat input impertinence (connected with the final rate of fuel drop).

During the process of fuel injection into the combustion chamber with the usage of the volumetric method of carburetion the fuel distribution by holes in a sprayer plays an important part. The sprayer usually has 4 and more holes. In the work process of manufacturing holes one can make the divergences of calcu-

\footnotetext{
${ }^{1}$ sle@agtu.secna.ru

${ }^{2}$ sae59@mail.ru

${ }^{3}$ alesc@newmail.ru
}

lated parameters: diameter, angles of arrangement and holes surface condition. Such divergences result in the change of the process of carburetion -fuel burning in the combustion chamber (high soot evolution, burning imperfection, etc). Parameter describing the effective section of outlet holes is $\mathrm{m}_{\mathrm{f}}$, the determination of which is brought to the measurement of the pressure drop and average fuel consumption [1].

\section{Mathematical model of the research of speed abi- lity of fuel dispersion}

Successful solution into the problems of decreasing fuel consumption, combustion efficiency, ecological compatibility, error-free performance of combustion engines requires developing a modem procedure of the analysis of the operating cycles efficiency based on fundamental laws of thermodynamics and special investigations of the intercylinder processes regularities.

According to Lambert-Ber law the relative intensity of the light radiation having passed through the medium is equal to

$$
J(t)=\frac{I(t)}{I_{O}}=\exp \{-\alpha n L\},
$$

here $\alpha$ is the optical factor of the fuel flow; $n$ is the particles concentration in the volume of the fuel flow pierced with the light radiation; $L$ is the flow thick- 
ness in the corresponding section.

Intensity and concentration of the fuel flow in section are equal to

$$
\begin{aligned}
& g(t)=\frac{N(t)}{t_{o}}, \\
& n(t)=\frac{N(t)}{V} .
\end{aligned}
$$

correspondently, here $N(t)$ is the quantity of particles in the flow volume pierced with the light radiation $V$; $n(t)$ is the particles concentration in this volume. The output signal $U(t)$ of the photo sensor is equal to

$$
U(t)=S I(t),
$$

here $S$ is the photo sensor sensitivity. Then

$$
\frac{U(t)}{U_{o}}=\frac{S I(t)}{S I_{o}}=J(t)=\exp \left\{-\frac{\alpha L g(t) t_{o}}{V}\right\}=\exp \{-Q g(t)\},(5)
$$

here $Q$ is the constant of the measurement unit conversion.

Mass of the pulsed dispersed flow $M$ having passed through the section of the flow for the time $t_{b}$ is equal to

$$
M=\int_{0}^{t_{b}} g(t) d t,
$$

here $t_{b}$ is the time from the beginning up to the end of the pulsed dispersed flow injection.

If the sections are at the close distance from each other then one can take the equality of the fuel mass having passed through the section, for the time of the flow development.

$$
M_{1}=M_{2} \text {. }
$$

Then

$$
\int_{o}^{t_{i}} \operatorname{Ln} U_{1}(t) d t=\int_{o}^{t_{i+1}} \operatorname{Ln} U_{2}(t) d t
$$

According to this procedure the relative mass of the fuel flow having passed through the chosen optical section is calculated, masses are compared, normalized and the time of the delay of the fuel mass transport from the first up to the second section is determined [2].

\section{Time-of-flight test bench for the determination of speed ability of atomized fuel}

Developed at Altai State University test bench allows one to measure the optical density of fuel flow developing through two sections series arranged. In every section there is the source of optical radiation light-emitting diode. Passing through the flow the light intensity changes according to the flow density and further it is registered with the photodiode. A signal from the photodiode intensifies, is converted into the digital form and is handled with the computer.

The scheme of the bench is shown in Fig 1. On the fuel bench Mirkez there is the pump of high pressure 2. The fuel proceeds under the pressure over pipeline 3 onto nozzle 5, placed on support 4. The sprayer of nozzle generates dispersed fuel flow 6 developing through sections 7 and 8 . Section includes the ring in which there are a light radiator and a photo sensor arranged opposite each other. From the photo sensor, the electric signal proceeds onto digital oscillograph 11 (C9-8) and further in a digital form onto computer 12. The dynamics of the pressure change is registered with strain sensor 9, the signal from which is processed with special strain-gauge amplifier 10 and is supplied onto oscillograph input 11 [3].

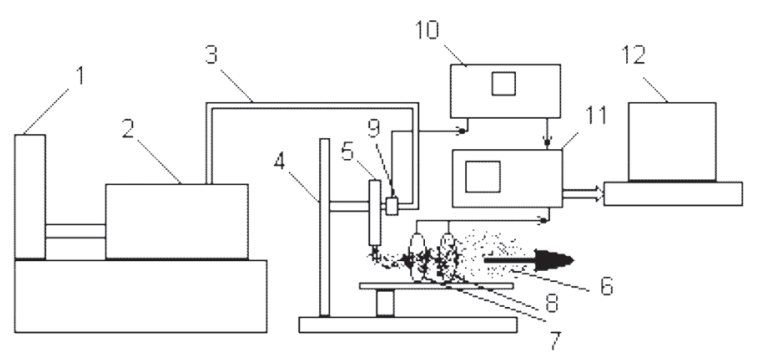

Fig 1. Scheme of the test bench for the determination of the fuel flow speed abilities

In Fig 2 the change of the optical density of the fuel flow is shown in sections at the distance of $5 \mathrm{~cm}$ and $10.3 \mathrm{~cm}$ from the sprayer point with 5 holes of $0,25 \mathrm{~mm}$ placed onto the nozzle FD-22.

The time is indicated by abscissa axis in msec, by coordinate axis the relative optical density is indicated in gradation of ACP. The time of the flow development in the first section is $3 \mathrm{msec}$, in the second one is about 4 msec [4].

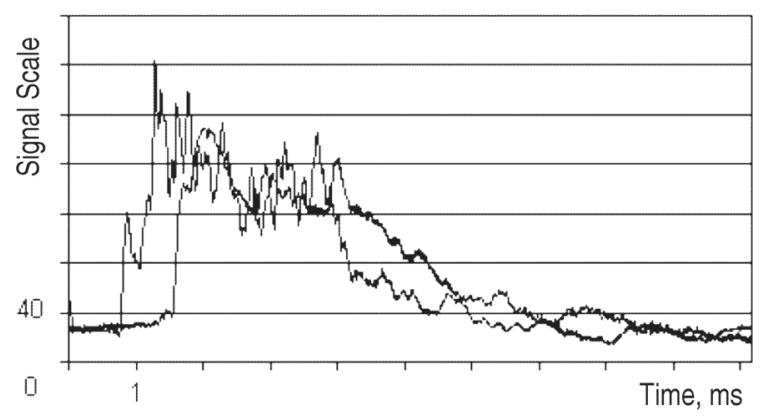

Fig 2. Change of the optical density of the fuel flow in section at the distance of 5 and $10,3 \mathrm{~cm}$ as a function of the sprayer point 
In Fig 3 the pressure of fuel is shown before the nozzle and up of the pin of a sprayer point. Experiments were carried out under atmospheric conditions (injection into the open space) with the rotation frequency of the fuel pump shaft of $500 \mathrm{~min}^{-1}$ and the fuel consumption of $92 \mathrm{~mm}^{3} /$ cycle.

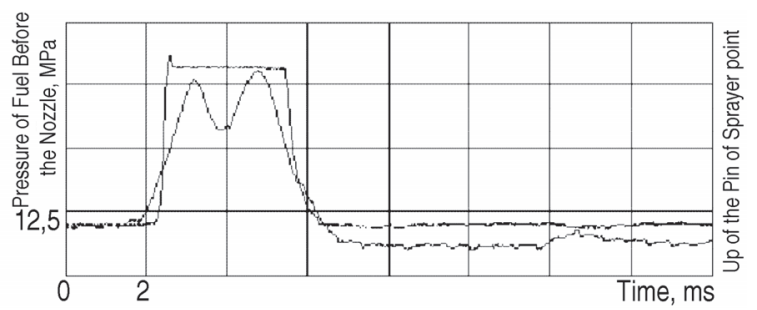

Fig 3. Change of the pressure of fuel before the nozzle and up of the pin of sprayer point

\section{Analysis of speed ability of fuel dispersion}

Having integrated the relative optical density by time according to the method of trapezoids we get the relative flow mass having passed through the chosen section Fig 4.

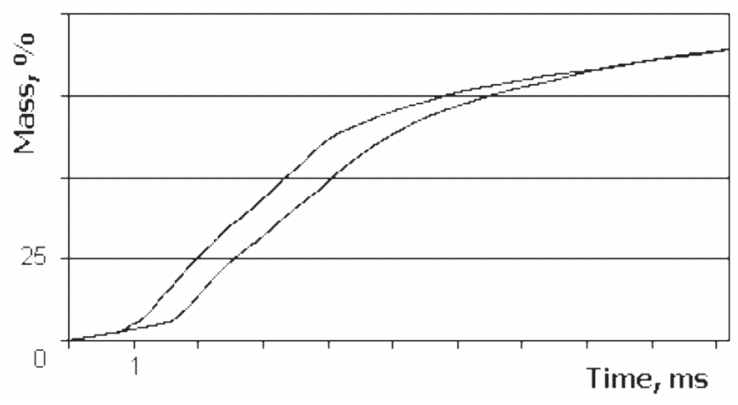

Fig 4. Time of the fuel mass delay between the sections

Knowing the delay time and the distance between the sections we get the bar chart of the mass distribution by the speed in the pulsed flow of the sprayed fuel (Fig 5).

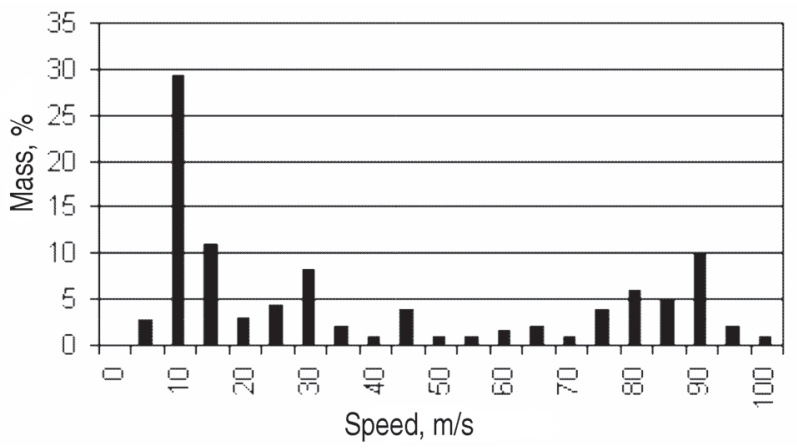

Fig 5. Fuel mass distribution by the speed in the chosen sections
Having determined the speed of the fuel movement it is possible to forecast the location of the main area of the fuel flow at the characteristic moment of the fuel ignition and burning that determines the process of burning and cracking.

In Fig 5 the speed of particles is shown in a band of measuring section which has a complicated character of distribution. The majority of particles being at the head of fuel flow having met the resistance of fixed medium has the speed of 10-20 m/s. The particles of fuel being at the end of flow can have high speeds, but their mass is less than at the head. The greater mass of particles at the head of fuel testifies the fuel concentration in this band. The increasing pressure of injection extra dispersion of fuel allows "to smooth" the bar chart of mass distribution, to reduce the concentration of particles at the head. Splash of speed at $100 \mathrm{~m} / \mathrm{s}$ can be determined by flow pulsation.

During the determination of fuel flow speed 5 series of injections were investigated. The results of the investigations are shown in Fig 6. The amount of time from the beginning of injection up to the change of the relative optical density in every channel up to 0,25 units was determined for each of 5 injections. The digitization frequency of the signal from the photo sensor was $200 \mathrm{kHz}$. Knowing the distance between the sections we examined the speed of flow for each of 5 injections. 5 injections were investigated for gyration of fuel pump 500, 650, 750, $850 \mathrm{~min}^{-1}$ and the calculation of middle speed was made which composed 35,$3 ; 79,8 ; 88,0 ; 90,6 \mathrm{~m} / \mathrm{s}$ correspondingly. The nonlinear growth of fuel flow speed in accordance with the increase of gyration of fuel pump is seen in Fig It is explained (as the authors consider) by the nonlinear character of increasing fuel pressure before the nozzle and the speed of fuel expiration from the hole in accordance with the growth of fuel pump gyration [5].

During the experiment the frequency of camshaft gyration of a fuel pump increased and as a result the speed of volumetric injection and pressure before the

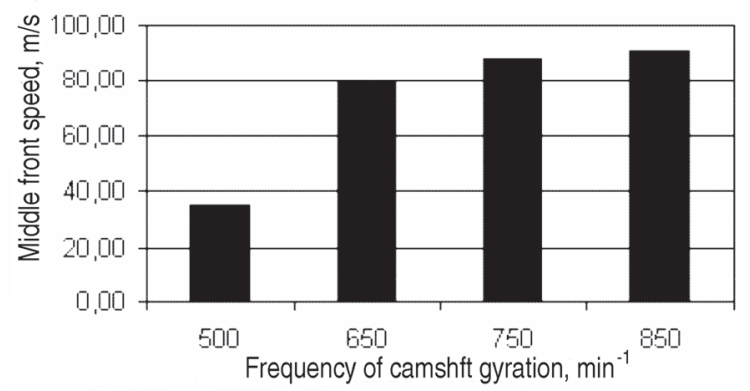

Fig 6. Change of the dependence of middle front speed of the fuel flow from the frequency of camshaft gyration 
nozzle increased too. In Fig 7 the experimentally obtained dependence of fuel pressure before the nozzle from the frequency of camshaft gyration is shown.

While increasing the frequency of camshaft gyration from 500 till $850 \mathrm{~min}^{-1}$ the maximal fuel pressure before the nozzle grew from 39,6 till 54,8 MPa (by $38 \%$ ) at the expense of ascending volumetric speed of fuel displacement by the plunger and lowering of leakages.

The experiments were made at the angle of $28^{\circ}$, fuel grade - diesel, fuel toughless at $293^{\circ} \mathrm{K}$ from 3,5 up to $6,0 \mathrm{~mm}^{2} / \mathrm{s}$.

Analyzing Fig 8 of particles distribution by speed obtained during the experiment it was has revealed that by the increase of gyration frequency with the growth of fuel pressure before the nozzle the change of particles speed of the fuel flow in dependence of mass is seen.

The pressure of fuel before the nozzle increased so as the amount of fuel mass, setting with high speeds (e.g. near $26 \%$ of fuel mass sets with the speed more than $50 \mathrm{~m} / \mathrm{s}$, and by the frequency of camshaft gyration $850 \mathrm{~min}^{-1}$ already $52 \%$ ).

\section{Conclusions}

The distribution of fuel mass by the speed will depend on the parameters of fuel expiration from the hole, the distance up to measuring sections properties of a fluid etc while observing the regime parameters the influence of constructive factors of the sprayer (divergence from the standard in work pro-

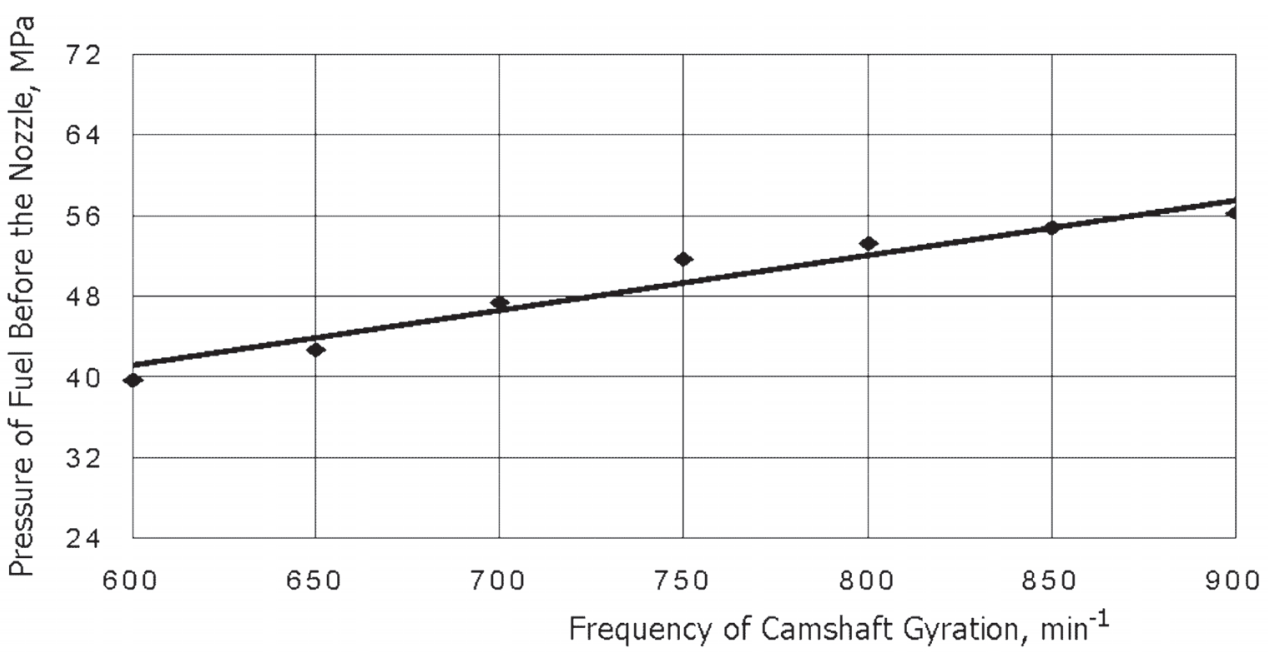

Fig 7. The dependence of middle pressure of fuel before the nozzle of the fuel flow from the frequency of camshaft gyration
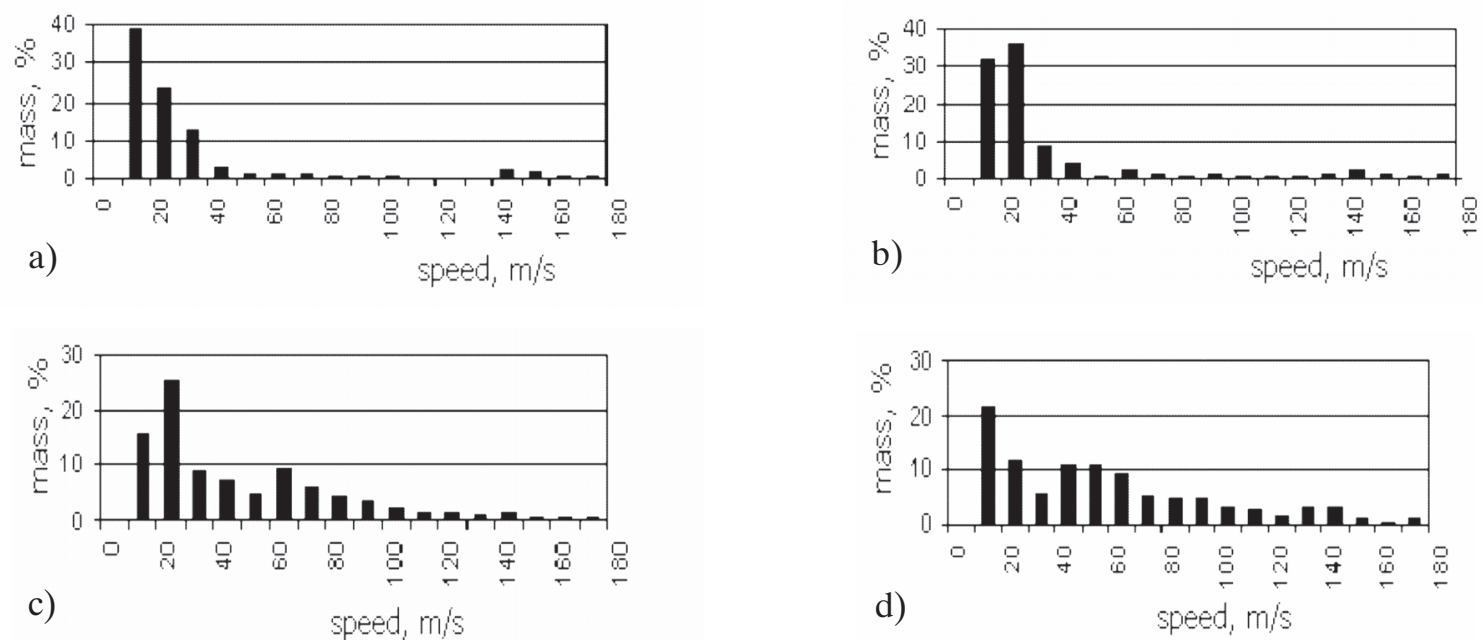

Fig 8. The distribution of fuel mass by the speed by the frequency of camshaft gyration: a) $500 \mathrm{~min}^{-1}$; b) $650 \mathrm{~min}^{-1}$; c) $750 \mathrm{~min}^{-1}$; d) $850 \mathrm{~min}^{-1}$ 
cess of manufacturing) can be determined. The procedure of the determination of speed abilities of the fuel flow gives the preconditions for creating the bench for the determination of fuel sprayers in work process of manufacturing with the aim of their division into the groups of one-type parameters. Such approach assumes the usage of manufacturing standard that is the sprayer and the fuel pump with the best ability of injection.

The experimental bench allows to investigate the process of fuel dispersion, the speed of mass and the change of pressure in a pipeline before the nozzle in a real time process and during different speeds of fuel pump gyration. In conclusion it is possible to note that optical methods of the determination of the process of fuel dispersion made for different types of sprayers can be used in a work process of manufacturing that allows to improve the quality of produced materials rejecting unqualitative sprayers.

Investigating relative optical density by every sprayer hole at the same cycle the correlation of fuel mass distribution by the holes can be determined.

Having investigated the process of dispersion by the increasing frequency of a fuel pump shows the increase of front speed and the development of fuel flow and allows to make the conclusion about the stability or the instability of the operation of the nozzle on these or those turnovers.

The further task of investigation concerns the process of working out standard criteria of optical abilities of fuel jets.

\section{References}

1. Sviridov, Yu. B. Generator of fuel jet and combustion in diesel engines (Смесеобразование и сгорание в дизелях). Leningrad: Machineindustry, 1972. 224 p. (in Russian).

2. Evstigneev, V. V.; Gulyaev, P. Yu.; Eskov, A. V. Method of the Speed Determination of the Pulsed Aerodispersed Flow (Способ определения скоростных характеристик импульсного аэродисперсного потока), 1998. Patent No 2147749 (in Russian).

3. Evstigneev, V. V.; Eskov, A. V.; Karpov, I. E.; Ognev, I. V. Test Bench of Fuel Sprayers. In: Proceed. of the Conf. (Труды 8-ой междунар. научно-практич. конф. студ., асп. и мол. ученых). Tomsk, TPU, 2002, p. 110 111 (in Russian).

4. Svistula, A. E.; Matievsky, D. D.; Gulaev, P. Yu.; Eskov, A. V. Experimental research of the characteristics of fuel sprays of diesel injectors. Engine Engineering (Двигателестроение), No 1, 1999, p. 28-31 (in Russian).

5. Matievsky, D. D.; Eskov, A. V.; Svistula, A. E.; Ognev, I. $V$. The research of recurrence and definition of speed of front of an impulse dispersible flow of liquid fuel. In: Proceed. of the Conf. (Труды междунар. научно-технич. конф., 23-25 апреля 2003 г.).Cheliabinsk, YyUrGU, 2003, p. 116-118 (in Russian). 